PROCEEDINGS OF THE

AMERICAN MATHEMATICAL SOCIETY

Volume 135, Number 10, October 2007, Pages 3133-3141

S 0002-9939(07)08731-X

Article electronically published on June 19, 2007

\title{
ON UNBOUNDEDNESS OF MAXIMAL OPERATORS FOR DIRECTIONAL HILBERT TRANSFORMS
}

\author{
G. A. KARAGULYAN
}

(Communicated by Michael T. Lacey)

\begin{abstract}
We show that for any infinite set of unit vectors $U$ in $\mathbb{R}^{2}$ the maximal operator defined by

$$
H_{U} f(x)=\sup _{u \in U}\left|\operatorname{pv} \int_{-\infty}^{\infty} \frac{f(x-t u)}{t} d t\right|, \quad x \in \mathbb{R}^{2},
$$
\end{abstract}

is not bounded in $L^{2}\left(\mathbb{R}^{2}\right)$.

\section{INTRODUCTION}

For a rapidly decreasing function $f$ and a unit vector $u=(\cos \theta, \sin \theta), \theta \in[0,2 \pi]$, we define

$$
H_{u} f(x, y)=\mathrm{pv} \int_{-\infty}^{\infty} \frac{f(x-t \cos \theta, y-t \sin \theta)}{t} d t
$$

which is the one-dimensional Hilbert transform along the direction $u$. It is well known that this operator can be extended to a bounded operator from $L^{p}\left(\mathbb{R}^{2}\right)$ to itself when $1<p<\infty$. In this paper we study operators

$$
H_{U} f(x, y)=\sup _{u \in U}\left|H_{u} f(x, y)\right|,
$$

where $U$ is a set of unit vectors $u$ in $\mathbb{R}^{2}$. Analogous operators for the maximal functions are properly investigated. The case of lacunary $U$ was first considered in the papers [18], 7], 15]. A final result was obtained by A. Nagel, E. M. Stein and S. Wainger in [15. They proved the boundedness of the norms of these operators in $L^{p}, 1<p<\infty$, for a lacunary $U$. Upper bounds of such operators depending on the cardinality $\# U$ of the set $U$ are considered in the papers [4, [5], 6], 13, [14, [19, and the definitive estimates are due to N. Katz (13], 14]), where he obtained a logarithmic order for the norms of two different maximal operators depending on $\# U$. Various generalizations of these results were considered in series of papers ([1], 2], 3], 10], [17]).

As for the operator (11) there were no results except for the bound

$$
\left\|H_{U} f(x)\right\|_{L^{2}} \lesssim \log \# U\|f\|_{L^{2}} .
$$

This is an immediate consequence of the Menshov-Rademacher theorem (see [9] or [12]), in spite of the fact that in Katz's theorems a subtle range of ideas are used.

Received by the editors February 21, 2006.

2000 Mathematics Subject Classification. Primary 42B25, 42B20.

Key words and phrases. Hilbert transform, maximal function.

(C)2007 American Mathematical Society Reverts to public domain 28 years from publication 
It was not even known whether or not $H_{U}$ is bounded in $L^{2}$ for an infinite lacunary set $U$. The main result of the paper is unboundedness of $H_{U}$ in $L^{2}$ for any infinite $U$.

Theorem 1. For any infinite set of unit vectors $U$ the operator $H_{U}$ cannot be extended to a bounded operator from $L^{2}\left(\mathbb{R}^{2}\right)$ to $L^{p}\left(\mathbb{R}^{2}\right)$ for all $1 \leq p<\infty$.

This theorem is an immediate consequence of the following estimate.

Theorem 2. If $U$ is a finite set and $1 \leq p<\infty$, then $\left\|H_{U}\right\|_{L^{2} \rightarrow L^{p}} \geq c \sqrt{\log \# U}$, where $c>0$ is an absolute constant.

The author is very grateful to M. T. Lacey for his hospitality during the two visits to Georgia Tech in 2003 and 2004, and for helpful conversations about these problems.

\section{Proof of the theorems}

Let $f_{n}(x), n=1,2, \cdots, 2^{m}-1\left(f_{n} \not \equiv 0\right)$, be a system of functions defined on the square

$$
Q=[-\pi, \pi] \times[-\pi, \pi] .
$$

In some places we shall use for $f_{n}(x)$ double numbering, defined by

$$
f_{j}^{(k)}(x)=f_{n}(x), \quad n=2^{k}+j-1, \quad 1 \leq j \leq 2^{k}, \quad k=0,1, \cdots, m-1 .
$$

We shall say the sequence $f_{n}(x)=f_{j}^{(k)}(x)$ is a tree-system, if

(2) $\operatorname{supp} f_{2 j-1}^{(k+1)} \subset\left\{x \in Q: f_{j}^{(k)}(x)>0\right\}, \quad \operatorname{supp} f_{2 j}^{(k+1)} \subset\left\{x \in Q: f_{j}^{(k)}(x)<0\right\}$.

Applying (2) several times we get

$$
\begin{aligned}
& \operatorname{supp} f_{i}^{(k+r)} \subset\left\{x \in Q: f_{j}^{(k)}(x)>0\right\} \quad \Leftrightarrow \quad i \in\left(2^{r} j-2^{r}, 2^{r} j-2^{r-1}\right], \\
& \operatorname{supp} f_{i}^{(k+r)} \subset\left\{x \in Q: f_{j}^{(k)}(x)<0\right\} \quad \Leftrightarrow \quad i \in\left(2^{r} j-2^{r-1}, 2^{r} j\right],
\end{aligned}
$$

and then

(3) $\operatorname{supp} f_{i}^{(k+r)} \cap\left\{x \in Q: f_{j}^{(k)}(x)>0\right\}=\varnothing \quad \Leftrightarrow \quad i \notin\left(2^{r} j-2^{r}, 2^{r} j-2^{r-1}\right]$,

(4) $\operatorname{supp} f_{i}^{(k+r)} \cap\left\{x \in Q: f_{j}^{(k)}(x)<0\right\}=\varnothing \quad \Leftrightarrow \quad i \notin\left(2^{r} j-2^{r-1}, 2^{r} j\right]$.

The following lemma for the Haar system has been proved by E. M. Nikishin and P. L. Ul'yanov [16, and we use the same idea to prove a general one (see also [12]).

Lemma 1. If $f_{n}(x), x \in Q, n=1,2, \cdots, 2^{m}-1$, is tree-system, then there exists a permutation $\sigma$ of the numbers $\left\{1,2, \cdots, 2^{m}-1\right\}$ such that

$$
\sup _{1 \leq l<2^{m}}\left|\sum_{n=1}^{l} f_{\sigma(n)}(x)\right| \geq \frac{1}{3} \sum_{n=1}^{2^{m}-1}\left|f_{n}(x)\right| .
$$

Proof. We connect with each $f_{n}(x), n=2^{k}+j-1$, a number

$$
t_{n}=\frac{2 j-1}{2^{k+1}} \in[0,1] \text {. }
$$

Note they are not equal for different $n$ 's. Define the permutation $\sigma$ so that

$$
t_{\sigma(1)}<t_{\sigma(2)}<\cdots<t_{\sigma\left(2^{m}-1\right)}
$$


We shall prove that for any $x \in Q$ there exists a number $l=l(x)$ with

$$
\begin{aligned}
& f_{\sigma(n)}(x) \geq 0, \text { if } n>l(x), \\
& f_{\sigma(n)}(x) \leq 0, \text { if } n \leq l(x) .
\end{aligned}
$$

Defining

$$
l=l(x)=\sup \left\{n: 1 \leq n<2^{m}, f_{\sigma(n)}(x) \leq 0\right\},
$$

we shall have (6) immediately and if $\nu=\sigma(l(x)+1)$, then

$$
f_{\nu}(x)>0 .
$$

To prove (5) it is enough to show that if (7) holds and $t_{n}>t_{\nu}$, then $f_{n}(x) \geq 0$. Suppose

$$
\nu=2^{k}+j-1, \quad n=2^{s}+i-1 .
$$

According to the assumption

$$
t_{\nu}=\frac{2 j-1}{2^{k+1}}<\frac{2 i-1}{2^{s+1}}=t_{n} .
$$

If $s>k$, then $s=k+r(r>0)$. From (8) we get

$$
i>2^{r} j-2^{r-1} \text {. }
$$

Therefore by (3) we obtain

$$
\operatorname{supp} f_{n} \cap\left\{f_{\nu}>0\right\}=\varnothing .
$$

Since $f_{\nu}(x)>0$ we get $f_{n}(x)=0$.

If $s<k$, then $k=s+r(r>0)$. Applying (8) we get

$$
j \leq 2^{r} i-2^{r-1} .
$$

Hence by (4) we have

$$
\operatorname{supp} f_{\nu} \cap\left\{f_{n}<0\right\}=\varnothing \text {. }
$$

By (7) we conclude $f_{n}(x) \geq 0$. So (5) and (6) are proved. Using them, we obtain

$$
\max _{1 \leq l<2^{m}}\left|\sum_{n=1}^{l} f_{\sigma(n)}(x)\right|=\max \left\{-\sum_{n=1}^{l(x)} f_{\sigma(n)}(x),\left|\sum_{n=1}^{2^{m}-1} f_{\sigma(n)}(x)\right|\right\} .
$$

If

$$
-\sum_{n=1}^{l(x)} f_{\sigma(n)}(x)=\sum_{n=1}^{l(x)}\left|f_{\sigma(n)}(x)\right|<\frac{1}{3} \sum_{n=1}^{2^{m}-1}\left|f_{\sigma(n)}(x)\right|,
$$

then we get

$$
\sum_{n=l(x)+1}^{2^{m}-1}\left|f_{\sigma(n)}(x)\right|>2 \sum_{n=1}^{l(x)}\left|f_{\sigma(n)}(x)\right|
$$

and therefore

$$
\begin{aligned}
\left|\sum_{n=1}^{2^{m}-1} f_{\sigma(n)}(x)\right| \geq\left|\sum_{n=l(x)+1}^{2^{m}-1} f_{\sigma(n)}(x)\right| & -\left|\sum_{n=1}^{l(x)} f_{\sigma(n)}(x)\right| \\
& >\frac{1}{2} \sum_{n=l(x)+1}^{2^{m}-1}\left|f_{\sigma(n)}(x)\right|>\frac{1}{3} \sum_{n=1}^{2^{m}-1}\left|f_{\sigma(n)}(x)\right| .
\end{aligned}
$$


Thus we conclude

$$
\sup _{1 \leq l<2^{m}}\left|\sum_{n=1}^{l} f_{\sigma(n)}(x)\right|>\frac{1}{3} \sum_{n=1}^{2^{m}-1}\left|f_{\sigma(n)}(x)\right|=\frac{1}{3} \sum_{n=1}^{2^{m}-1}\left|f_{n}(x)\right| .
$$

Fix a Schwartz function $\phi(x)$ with

$$
\phi(x)>0, \quad \int_{\mathbb{R}} \phi(x) d x=1, \quad \operatorname{supp} \widehat{\phi} \subset[-1,1] .
$$

We consider operators

$$
\Phi_{n}(f)=\Phi_{n}(x, y, f)=n^{2} \int_{\mathbb{R}} f(x-t, y-s) \phi(n t) \phi(n s) d t d s, \quad n=1,2, \cdots
$$

Applying (9), for any $f \in L^{\infty}\left(\mathbb{R}^{2}\right)$ we get

$$
\begin{gathered}
\inf _{(x, y) \in \mathbb{R}^{2}} f(x, y) \leq \Phi_{n}(x, y, f) \leq \sup _{(x, y) \in \mathbb{R}^{2}} f(x, y), \\
\operatorname{supp} \widehat{\Phi_{n}(f)} \subset[-n, n] \times[-n, n] .
\end{gathered}
$$

If in addition $f$ is compactly supported, using a standard argument, we conclude

$$
\left\|\Phi_{n}(f)-f\right\|_{L^{2}} \rightarrow 0 \text {, as } n \rightarrow \infty .
$$

If

$$
n=2^{k}+j-1, \quad 1 \leq j \leq 2^{k}, \quad k=0,1, \cdots, m-1,
$$

then we denote

$$
\bar{n}=2^{k-1}+\left[\frac{j+1}{2}\right]
$$

where $[\cdot]$ means an integer part of a number. Using this notation we may write the conditions (2) by

$$
\operatorname{supp} f_{n} \subset\left\{(-1)^{j+1} \cdot f_{\bar{n}}>0\right\} .
$$

We shall consider sectors defined by

$$
\left\{(x, y) \in \mathbb{R}^{2}: x+i y=r e^{i \theta}, r \geq 0, \alpha \leq \theta \leq \beta\right\}
$$

where $0 \leq \alpha<\beta \leq 2 \pi$. Some arguments in the proof of following lemma are derived from the paper [11.

Lemma 2. Let $S_{n}, n=1,2, \cdots, \nu=2^{m}-1$, be sectors on the plane. Then there exist functions $f_{n} \in L^{2}\left(\mathbb{R}^{2}\right), n=1,2, \cdots, \nu$, such that

$$
\begin{gathered}
\operatorname{supp} \widehat{f_{n}} \subset S_{n}, \\
\sum_{j=1}^{\nu}\left\|f_{j}\right\|_{L^{2}}^{2} \leq c_{1}, \\
\left|\left\{(x, y) \in Q: \max _{1 \leq n \leq \nu}\left|\sum_{j=1}^{n} f_{\sigma(j)}(x, y)\right|>c_{3} \sqrt{\log \nu}\right\}\right|>c_{2},
\end{gathered}
$$

where $\sigma$ is the permutation from Lemma 1 and all the constants are absolute.

Proof. We will assume (14) everywhere below. For a given $\varepsilon>0$ define sets $E_{n}=$ $E_{j}^{(k)} \subset Q\left(E_{1}=E_{1}^{(0)}=Q\right), g_{n} \in L^{\infty}\left(\mathbb{R}^{2}\right)$ and $p_{n}, q_{n} \in \mathbb{Z}$ with conditions

a) $E_{n}=\left\{(x, y) \in E_{\bar{n}}:(-1)^{j+1} \cos \left(p_{\bar{n}} x+q_{\bar{n}} y\right)>0\right\}, n=2,3, \cdots, \nu$,

b) $0 \leq g_{n} \leq 1,\left\|g_{n}-\mathbb{I}_{E_{n}}\right\|_{L^{2}} \leq \varepsilon, n=1,2, \cdots, \nu$,

c) $\operatorname{supp} \widehat{g}_{n} \subset S_{n}-\left(p_{n}, q_{n}\right), n=1,2, \cdots, \nu$,

d) $\int_{E_{n}}\left|\cos \left(p_{n} x+q_{n} y\right)\right| d x d y>\frac{\left|E_{n}\right|}{3}$. 
We do it by induction. Take $E_{1}=E_{1}^{(0)}=Q$. According to (13) there exists $l>0$ with

$$
\left\|\Phi_{l}\left(\mathbb{I}_{E_{1}}\right)-\mathbb{I}_{E_{1}}\right\|_{L^{2}}<\varepsilon
$$

Define $g_{1}=\Phi_{l}\left(\mathbb{I}_{E_{1}}\right)$ and then applying (11) we get b) for $n=1$. We note that if $E$ is a measurable set then

$$
\begin{aligned}
\int_{E}|\cos (p x+q y)| d x d y \geq \int_{E} \cos ^{2}(p x+q y) d x d y \\
=\frac{|E|}{2}+\int_{E} \frac{\cos (2(p x+q y))}{2} d x d y \rightarrow \frac{|E|}{2} \text { as }|p|,|q| \rightarrow \infty .
\end{aligned}
$$

This observation shows that for sufficiently large $p_{1}=p$ and $q_{1}=q$ we shall have condition d) for $n=1$. On the other hand by (12) supp $\widehat{g}_{1}$ is bounded. Thus for an appropriate $p_{1}, q_{1}$ we will have also c) (with $n=1$ ). Certainly we can choose $p_{1}$ and $q_{1}$ common for both conditions c) and d). Now we suppose that the conditions a)-d) hold for any $k<n$, in particular for $\bar{n}$. We define $E_{n}$ by the equality in a). Then we choose the positive integer $l$ with

$$
\left\|\Phi_{l}\left(\mathbb{I}_{E_{n}}\right)-\mathbb{I}_{E_{n}}\right\|_{L^{2}}<\varepsilon
$$

and denote $g_{n}=\Phi_{l}\left(\mathbb{I}_{E_{n}}\right)$. Again applying (19) and using the boundedness of $\operatorname{supp} \widehat{g}_{n}$ we may choose integers $p_{n}, q_{n}$ satisfying c) and d) together. Using condition a), it is easy to check that the sets $E_{n}$ satisfies the conditions

$$
\begin{gathered}
E_{j}^{(k)} \cap E_{j^{\prime}}^{(k)}=\varnothing, \text { if } j \neq j^{\prime}, \\
E_{2 j-1}^{(k+1)} \cup E_{2 j}^{(k+1)} \subset E_{j}^{(k)}, \quad\left|E_{j}^{(k)} \backslash\left(E_{2 j-1}^{(k+1)} \cup E_{2 j}^{(k+1)}\right)\right|=0 .
\end{gathered}
$$

Using this properties we conclude

$$
\sum_{n=1}^{\nu} \mathbb{I}_{E_{n}}(x, y)=m, \text { almost everywhere on } Q .
$$

Now we define

$$
f_{n}(x, y)=\frac{e^{i\left(p_{n} x+q_{n} y\right)} g_{n}(x, y)}{\sqrt{m}} .
$$

Condition (16) immediately follows from c), because

$$
\operatorname{supp} \widehat{f}_{n}=\operatorname{supp} \widehat{g}_{n}+\left(p_{n}, q_{n}\right) \text {. }
$$

On the other hand taking a small $\varepsilon$ by b) and (20) we obtain

$$
\sum_{n=1}^{\nu} \int_{Q}\left|f_{n}\right|^{2}=\frac{1}{m} \sum_{n=1}^{\nu} \int_{Q}\left|g_{n}\right|^{2} \leq \frac{2 \nu}{m} \varepsilon^{2}+\frac{2}{m} \sum_{n=1}^{\nu} \int_{Q} \mathbb{I}_{E_{n}} \leq c_{1},
$$

which gives (17). Now consider functions

$$
\tilde{f}_{n}=\operatorname{Re} f_{n} \cdot \mathbb{I}_{E_{n}}=\frac{\cos \left(p_{n} x+q_{n} y\right) \cdot g_{n}(x, y) \cdot \mathbb{I}_{E_{n}}(x, y)}{\sqrt{m}} .
$$

Applying b) and (21) we get

$$
\left\|\tilde{f}_{n}-\operatorname{Re} f_{n}\right\|_{L^{2}}^{2}=\int_{\mathbb{R}^{2} \backslash E_{n}}\left|\operatorname{Re} f_{n}\right|^{2} \leq \int_{\mathbb{R}^{2} \backslash E_{n}}\left|f_{n}\right|^{2}=\frac{1}{m} \int_{\mathbb{R}^{2} \backslash E_{n}}\left|g_{n}\right|^{2} \leq \frac{\varepsilon^{2}}{m} .
$$


On the other hand we have

$$
\begin{aligned}
\max _{1 \leq n \leq N}\left|\sum_{j=1}^{n} f_{\sigma(j)}\right| & \geq \max _{1 \leq n \leq N}\left|\sum_{j=1}^{n} \operatorname{Re} f_{\sigma(j)}\right| \\
& \geq \max _{1 \leq n \leq N}\left|\sum_{j=1}^{n} \tilde{f}_{\sigma(j)}\right|-\sum_{j=1}^{\nu}\left|\tilde{f}_{j}-\operatorname{Re} f_{j}\right| .
\end{aligned}
$$

From (23) we obtain

$$
\left\|\sum_{j=1}^{\nu}\left|\tilde{f}_{j}-\operatorname{Re} f_{j}\right|\right\|_{L^{2}} \leq \frac{\nu \varepsilon}{\sqrt{m}} .
$$

Therefore taking a small $\varepsilon>0$ we can say that

$$
\left|\left\{(x, y) \in Q: \sum_{j=1}^{\nu}\left|\tilde{f}_{j}-\operatorname{Re} f_{j}\right|>1\right\}\right| \leq \delta,
$$

for any given $\delta>0$. From (24) and (25) we conclude that to prove (18) and also the lemma, it is enough to prove

$$
\left|\left\{(x, y) \in Q: \max _{1 \leq n \leq \nu}\left|\sum_{j=1}^{n} \tilde{f}_{\sigma(j)}(x, y)\right|>c_{3} \sqrt{\log \nu}\right\}\right|>c_{2} .
$$

Let us show that $\tilde{f}_{n}$ is a tree-system, i.e. it satisfies (15). Since $g_{n}>0$ from (22) we get that $\tilde{f}_{n}(x, y)$ and $\cos \left(p_{n} x+q_{n} y\right)$ have same sign in the set $E_{n}$. Therefore by a) we obtain

$$
\begin{aligned}
& \operatorname{supp} \tilde{f}_{n} \subset E_{n}=\left\{(x, y) \in E_{\bar{n}}:(-1)^{j+1} \cos \left(p_{\bar{n}} x+q_{\bar{n}} y\right)>0\right\} \\
& \begin{aligned}
=\left\{(x, y) \in E_{\bar{n}}:(-1)^{j+1} \tilde{f}_{\bar{n}}(x, y)>0\right\}= & \left\{(x, y) \in E_{\bar{n}}:(-1)^{j+1} \tilde{f}_{\bar{n}}(x, y)>0\right\} \\
& =\left\{(x, y) \in Q:(-1)^{j+1} \tilde{f}_{\bar{n}}(x, y)>0\right\} .
\end{aligned}
\end{aligned}
$$

Hence $\tilde{f}_{n}$ is a tree-system. So according to Lemma 1 we have

$$
\max _{1 \leq n \leq N}\left|\sum_{j=1}^{n} \tilde{f}_{\sigma(j)}(x, y) \geq \frac{1}{3} \sum_{j=1}^{\nu}\right| \tilde{f}_{j}(x, y) \mid .
$$

From (21) and the conditions b) and d) we get

$$
\begin{array}{r}
\int_{Q}\left|\tilde{f}_{n}\right|=\frac{1}{\sqrt{m}} \int_{E_{n}}\left|g_{n} \cos \left(p_{n} x+q_{n} y\right)\right| d x d y \geq \frac{1}{\sqrt{m}} \int_{E_{n}}\left|\cos \left(p_{n} x+q_{n} y\right)\right| d x d y \\
-\frac{1}{\sqrt{m}} \int_{E_{n}}\left|\left(1-g_{n}\right) \cos \left(p_{n} x+q_{n} y\right)\right| d x d y \geq \frac{\left|E_{n}\right|}{3 \sqrt{m}}-\frac{\varepsilon}{\sqrt{m}} .
\end{array}
$$

If we take $\varepsilon>0$ to be small, then from (20) we obtain

$$
\int_{Q} \sum_{j=1}^{\nu}\left|\tilde{f}_{j}\right| \geq \frac{1}{3 \sqrt{m}} \sum_{n=1}^{\nu}\left|E_{n}\right|-\frac{\nu \varepsilon}{\sqrt{m}} \gtrsim \sqrt{m} .
$$

Combining this and (27) we get

$$
\int_{Q} \max _{1 \leq n \leq N}\left|\sum_{j=1}^{n} \tilde{f}_{\sigma(j)}(x, y)\right| \gtrsim \sqrt{m} .
$$


On the other hand by (20), (21) and b) for any $(x, y) \in Q$ we have

$$
\max _{1 \leq n \leq N}\left|\sum_{j=1}^{n} \tilde{f}_{\sigma(j)}(x, y)\right| \leq \sum_{j=1}^{\nu}\left|\tilde{f}_{j}(x, y)\right| \leq \frac{1}{\sqrt{m}} \sum_{j=1}^{\nu} \mathbb{I}_{E_{n}}(x, y) \leq \sqrt{m} .
$$

From (28) and (29) follows (26).

Proof of Theorem 2. For any region $S \subset \mathbb{R}^{2}$ we denote

$$
T_{S} f(x, y)=\int_{S} e^{i(\xi x+\eta y)} \widehat{f}(\xi, \eta) d \xi d \eta .
$$

Since the multiplier for the Hilbert transform is $i \cdot \operatorname{sign} x$, for any direction $u=$ $(\cos \theta, \sin \theta)$ we have

$$
\widehat{H_{u}} f(x, y)=i \cdot \operatorname{sign}(x \cos \theta+y \sin \theta) \widehat{f}(x, y) .
$$

Thus we conclude

$$
H_{u} f=i\left(2 \cdot T_{\Gamma_{u}} f-f\right)
$$

where

$$
\Gamma_{u}=\left\{(x, y) \in \mathbb{R}^{2}: x \cos \theta+y \sin \theta \geq 0\right\}
$$

Denote

$$
T_{U} f=\sup _{u \in U}\left|T_{\Gamma_{u}} f\right| .
$$

Let $U=\left\{u_{k}=\left(\cos \theta_{k}, \sin \theta_{k}\right): k=1,2, \cdots, N\right\}$ be the set of directions from Theorem 2. Without loss of generality we can assume $\theta_{k} \in(0, \pi / 2), \theta_{1}<\theta_{2}<$ $\cdots<\theta_{N}$ and $N=2^{m}$. According to (30), to prove Theorem 2 it is enough to prove that

$$
\left\|T_{U} f\right\|_{L^{1}} \gtrsim \sqrt{\log N}\|f\|_{L^{2}}
$$

for some function $f \in L^{2}\left(\mathbb{R}^{2}\right)$. We denote by $S_{k}, k=1,2, \cdots, \nu=2^{m}-1$, the sectors obtained by the vectors $\left(u_{k}\right)^{\perp}=\left(\cos \theta_{k},-\sin \theta_{k}\right)$ and $\left(u_{k+1}\right)^{\perp}=$ $\left(\cos \theta_{k+1},-\sin \theta_{k+1}\right)$, i.e.

(32) $S_{k}=\left\{(x, y) \in \mathbb{R}^{2}: x \geq 0, x \cos \theta_{k}+y \sin \theta_{k} \geq 0, x \cos \theta_{k+1}+y \sin \theta_{k+1} \leq 0\right\}$.

Hence if we suppose

$$
\operatorname{supp} \hat{f} \subset \bigcup_{k=1}^{\nu} S_{k}
$$

then we can write

$$
T_{U} f(x, y)=\sup _{1 \leq l \leq \nu}\left|\sum_{k=1}^{l} T_{S_{k}} f(x, y)\right| .
$$

We define functions $f_{n}$ satisfying the conditions of Lemma 2 corresponding to the sectors $S_{n}^{\prime}=S_{\sigma^{-1}(n)}$ in (32). Denote

$$
f=\sum_{k=1}^{\nu} f_{k}
$$

Since $S_{n}$ are mutually disjoint the functions $f_{n}$ are orthogonal. Thus by (17) we get $\|f\|_{L^{2}} \leq c_{1}$. From (16) we have supp $\widehat{f}_{n} \subset S_{\sigma^{-1}(n)}, n=1,2, \cdots, \nu$, i.e. supp $\widehat{f}_{\sigma(n)} \subset S_{n}$ and therefore

$$
T_{S_{n}} f(x)=f_{n}(x) .
$$


According to (33) we obtain

$$
T_{U} f(x, y)=\max _{1 \leq l \leq N}\left|\sum_{j=1}^{l} f_{\sigma(j)}(x, y)\right| .
$$

Using (17) and (18), we get

$$
\left|\left\{(x, y) \in Q: T_{U} f(x, y)>c_{3} \sqrt{\log \nu}\right\}\right|>c_{2},
$$

and therefore $\left\|T_{U} f\right\|_{L^{p}} \gtrsim \sqrt{\log N}\|f\|_{L^{2}}$.

\section{REFERENCES}

1. A.Alfonseca, Strong type inequalities and an almost-orthogonality principle for families of maximal operators along directions in $\mathbb{R}^{2}$, J. London Math. Soc., 67, 2003, No. 1, 208-218. MR.1942421 (2003j:42015)

2. A.Alfonseca, F.Soria and A.Vargas, A remark on maximal operators along directions in $\mathbb{R}^{2}$, Math. Res. Lett., 10, 2003, No. 1, 41-49. MR1960122(2004j:42010)

3. A.Alfonseca, F.Soria and A.Vargas, An almost-orthogonality principle in $L^{2}$ for directional maximal functions, Contemp. Math, 2003. MR1979927 (2004d:42026)

4. J.Barrionuevo, Estimates for some Kakeya-type maximal operators, Trans. Amer. Math. Soc., 335, 1993, No. 2, 667-682 MR1150012 (93f:42038)

5. J.Barrionuevo, A note on the Kakeya maximal operator, Math. Res. Letters, 3, 1996, No. 1, 61-65. MR.1393383 (98k:42023)

6. A. Córdoba, The Kakeya maximal function and the spherical summation multipliers, Amer. J. Math., 99, 1977, No. 1, 1-22. MR0447949 (56:6259)

7. A.Córdaba and R.Fefferman, On differentiation of integrals, Proc. Nat. Acad. of Sci. USA, 74, 1977, No. 6, 2211-2213. MR0476977 (57:16522)

8. A.Córdaba and R.Fefferman, On the equivalence between the boundedness of certain classes of maximal and multiplier operators in Fourier analysis, Proc. Nat. Acad. Sci. U.S.A., 74, 1977, No. 2, 423-425. MR0433117(55:6096)

9. S. Kaczmarz and H. Steinhaus, Theorie der Orthogonalreihen, PWN, Warsaw, 1935.

10. G.A.Karagulyan and M.T.Lacey, An estimate of the maximal operators associated with generalized lacunary sets, Journal Contemporary Math. Anal., 2004, Vol. 39, No. 1. MR2168200 (2006d:42021)

11. G.A.Karagulyan, On the selection convergence subsystems with lacunary density of numbers from an arbitrary orthonormal systems, Dokl. AN of Armenia, 1987, Vol. 84, No. 1, 17-20 (in Russian). MR0890822 (89a:42033)

12. B.S.Kashin and A.A.Saakian, Orthogonal series, AMS, Providence, R.I., 1989. MR1007141 (90g:42001)

13. N.H.Katz, Remarks on maximal operators over arbitrary sets of directions, Bull. London Math. Soc., 31, 1999, No. 6, 700-710. MR1711029 (2001g:42041)

14. N.H.Katz, Maximal operators over arbitrary sets of directions, Proc. Nat. Acad. Sci. U.S.A., 75, 1978, No. 3, 1060-1062. MR1681088 (2000a:42036)

15. A.Nagel, E.M.Stein and S.Wainger, Differentiation in lacunary directions, Duke Math. J., 97, 1979, No. 1, 67-79. MR0466470 (57:6349)

16. E.M.Nikishin and Ul'yanov, On absolute and unconditional convergence, Uspechi Math. Nauk., 22, 1967, No. 3, pp. 240-242 (in Russian). MR0212488 (35:3359)

17. P.Sjogren and P.Sjolin, Littlewood-Paley decompositions and Fourier multipliers with singularities on certain sets, Ann. Inst. Fourier (Grenoble), 31, 1981, No. 1, 157-175. MR0613033 (82g:42014)

18. J.-O.Strömberg, Weak estimates for maximal functions with rectangles in certain directions, Arkiv. for Mat, 15, 1978, 229-240. MR0487260 (58:6911)

19. J.-O.Strömberg, Maximal functions associated to rectangles with uniformaly distributed directions, Ann. of Math., 107, 1976, 399-402. MR0481883(58:1978)

20. A.Vargas, A remark on a maximal function over a Cantor set of directions, Rend. Circ. Mat. Palermo (2), 44, 1995, No. 2, 273-282. MR.1355485 (96m:42033) 
Institute of Mathematics, Armenian National Academy of Sciences, Marshal Baghramian aVe. 24B, Yerevan, 375019, Armenia

Current address: Department of Applied Mathematics, Yerevan State University, Yerevan, Armenia

E-mail address: karagul@instmath.sci.am 